\title{
Helping kids and youth in times of emotional crisis
}

\section{Daniel Grigat, Monique Fernquist, Andrea Allen, Tanya Drescher, Andrew Fisher, Katelyn Wieringa, Heather Hair, Marni Bercov}

Background: In 2017 the Emergency Strategic Clinical Network (ESCN) and Addiction and Mental Health Strategic Clinical Network (AMH SCN) of Alberta Health Services (AHS) launched a survey to understand the experience of young people seeking help in an Emergency Department (ED) for a mental health concern. Over 1500 surveys were completed, and qualitative results were analyzed thematically and interpreted in partnership with focus groups. A major theme of the responses was the feeling of being stigmatized when seeking help for a mental health concern, and that health care professionals' understanding, empathy, and competencies with youth mental health needs to be improved. In response to this finding, a nurse education workshop has been developed and is being piloted and evaluated.

Implementation: The ESCN and the AMH SCN are working together to develop, pilot, and evaluate new training for ED nurses. The education will debunk common misperceptions and associated stigma about the causes of mental illness and addictions by presenting the medical paradigm of a physical illness with a complex etiology including genetic and environmental influences. The objective of these workshops is that nurses receive training to increase knowledge of the science and determinants of addiction and mental health issues to enable them to provide care that is compassionate and trauma informed. Pilot workshops have been delivered to a lived-experience advisory committee, as well as four cohorts of ED nurses. The impact of the workshops is being evaluated to inform further implementation.

Evaluation Methods: Nurses participating in the pilots will complete a 10 question survey to establish a baseline of their knowledge, compassion, and confidence. This survey has been adapted from the Mental Health Commission of Canada Healthcare Providers Questionnaire, and the Attitudes Towards Child Mental Health Questionnaire. Upon completion of the workshop nurses are be asked to complete a second brief survey reflecting on how this training will impact their clinical practice, and the barriers to practice change. Finally, 90-days post-training nurses will be re-administered the 10 question baseline survey. In addition, they will be asked if their goals for clinical practice change have been achieved.

Results: Four cohorts consisting of 39 emergency department nurses have completed the workshop. All 39 nurses completed the baseline survey and the workshop survey.

The baseline survey results were that $90 \%$ of respondents agreed that mental illness and addiction deserves as much attention as physical illness, and 92\% agreed that healthcare providers need to advocate for people with mental illness and addiction. However, $79 \%$ of respondents reported that they were more comfortable treating physical illness than mental illness or addiction. Further, while $95 \%$ of respondents disagreed that mental illness was the result of a weak personality, only $74 \%$ disagreed that substance abusers have no self-control. Finally, when asked if adverse social circumstances can result in mental illness or addiction $8 \%$ of respondents disagreed and $49 \%$ were unsure. 
Following the workshop $100 \%$ of respondents agreed that the workshop was applicable to their clinical practice, and 92\% agreed that they understood better how to support these patients. Important themes arising from the qualitative questions were the need to show more empathy and compassion, and the realization that although an ED nurse cannot resolve an underlying mental illness or addiction they do have an important role to play in stabilizing an acute crisis.

\section{Advice and Lessons Learned}

1) Youth and their caregivers often do not know where to go in a time of emotional crisis. Their experience in the emergency department can be a source of further distress, and presents an opportunity to provide compassionate and trauma informed care.

2) Approaching the topic of improving care in the ED requires the involvement of multiple stakeholders, including emergency operational and strategic leadership, addiction and mental health specialists, front line clinicians, and the youth and caregivers receiving care.

3) A major barrier to providing higher quality care for youth experiencing an emotional crisis is knowledge of how to assess these patients and communicate them, as well as knowledge of and access to appropriate resources that can be leveraged in patient care. 